\title{
An Algorithm Informed by the Parathyroid Hormone Level Reduces Hypocalcemic Complications of Thyroidectomy
}

\author{
James E. Wiseman • Matthew Mossanen • \\ Philip H. G. Ituarte · Jonathan M. T. Bath • \\ Michael W. Yeh
}

Published online: 5 January 2010

(C) The Author(s) 2010. This article is published with open access at Springerlink.com

\begin{abstract}
Background Measurement of the parathyroid hormone (PTH) level following total thyroidectomy (TTx) may allow prediction of postoperative hypocalcemia. We present an algorithmic method of managing hypocalcemia preemptively, based on the PTH level $1 \mathrm{~h}$ after operation.

Materials and methods We examined 423 consecutive patients undergoing TTx at a single institution. A subset of patients were managed using an algorithm involving routine postoperative oral calcium administration and the early addition of oral calcitriol in patients with a low 1-h postoperative PTH level. Algorithm patients were compared to a concurrent, conventionally managed group. Outcomes measured included serum calcium levels, symptoms of hypocalcemia, postoperative complications, and receipt of intravenous (IV) calcium.

Results The algorithm was applied in 135 patients, and 288 patients were managed conventionally. Critically low calcium levels (total calcium $<7.5 \mathrm{mg} / \mathrm{dl}[1.88 \mathrm{mmol} / \mathrm{l}]$ or ionized calcium $<0.94 \mathrm{mmol} / \mathrm{l})$ were less common in algorithm patients $(10.6 \%$ vs. $25.3 \% ; p<0.005)$. Much of this difference was attributable to the protective impact of the algorithm on patients undergoing TTx for cancer, 30\% of whom developed critically low calcium levels when managed conventionally. Among patients requiring IV calcium, algorithm patients received fewer doses (1.29 vs.
\end{abstract}

J. E. Wiseman $(\bowtie) \cdot$ M. Mossanen · P. H. G. Ituarte .

J. M. T. Bath · M. W. Yeh

Endocrine Surgical Unit, Department of Surgery,

University of California Los Angeles, 10833 Le Conte Avenue,

72-229 CHS, Los Angeles, CA 90095, USA

e-mail: jwiseman@mednet.ucla.edu
1.86; $p<0.05)$. Low 1-h PTH levels were found in $21 \%$ (28/133) of algorithm patients, but these did not correlate with low calcium levels, suggesting that the algorithm compensated adequately for temporary hypoparathyroidism. No patients developed hypercalcemia.

Conclusions An algorithmic approach incorporating early postoperative PTH levels and routine administration of oral calcium reduces the risk of severe hypocalcemia after total thyroidectomy.

\section{Introduction}

The management of hypocalcemia after total thyroidectomy (TTx) remains a challenge. Transient hypocalcemia is one of the most common postoperative complications of TTx, occurring in $17-26 \%$ of patients, and it results from direct injury to, devascularization of, or accidental removal of the parathyroid glands [1-4]. Though early postoperative hypocalcemia is reversible in the great majority of cases, permanent hypoparathyroidism is likely much more common than previously suspected [1].

To manage postoperative hypocalcemia, most practitioners obtain serial serum calcium measurements and respond appropriately to low levels. Recently, several groups have advocated measurement of the parathyroid hormone (PTH) level several hours after operation in order to predict the development of hypocalcemia [5-13]. This practice has not been widely adopted, nor has it been incorporated into a standardized system of care for patients undergoing TTx.

In the present study, we examined the impact of a management algorithm incorporating the postoperative PTH level as a means of preempting the development of hypocalcemia after TTx. 


\section{Materials and methods}

Patient selection

Data on 476 consecutive patients undergoing total thyroidectomy at UCLA Medical Center between January 2006 and December 2008 were used in this retrospective cohort study. Patients with a history of hyperparathyroidism, kidney transplantation, or renal failure were excluded, as were patients undergoing emergency operations. In addition, patients with a history of previous thyroid surgery, those with nonthyroid cancers (namely cancers invading or metastasizing to the thyroid), and those undergoing simultaneous operations on the thymus, thyroglossal duct, or radical/modified radical lymph node dissection were excluded. Using these criteria, 421 patients were included in the final study group. All subjects were over the age of 18 .

An algorithm for the management of post-thyroidectomy calcium was implemented beginning on January 1, 2006 by select physicians in the Division of General Surgery (Fig. 1). This algorithm was developed at UCLA Medical Center and entails routine postoperative oral calcium supplementation for all patients, plus the early addition of calcitriol for patients with PTH levels $\leq 15 \mathrm{pg} / \mathrm{ml}$ (normal: $11-51 \mathrm{pg} / \mathrm{ml}$ ) at $1 \mathrm{~h}$ after surgery. Standardized, preprinted order sheets were created for algorithm implementation, which was carried out in conjunction with education of residents, nurses, and ancillary staff to ensure compliance.
Data collection

Demographic data collected included age, gender, and race. Co-morbid conditions were defined using the Deyo modification [9] of the Charlson co-morbidity index, which uses International Classification of Diseases, Ninth Revision (ICD-9-CM) codes from medical records to sum the number of conditions from 17 diagnostic categories. Other variables recorded included length of stay (LOS), number of days spent in the ICU, and number of doses of intravenous (IV) calcium received. The aggregate postoperative complication rate was determined using ICD-9-CM codes for six conditions: respiratory failure, hematoma formation, recurrent laryngeal nerve injury, wound complications, and signs or symptoms of hypocalcemia. Since complications were uncommon, a dichotomous variable for presence of any complications (present/absent) was used in some analyses. For algorithm patients, a single postoperative calcium level was measured from blood drawn at 4:00 a.m. on postoperative day 1 , whereas non-algorithm patients underwent serial serum calcium measurements every 6$8 \mathrm{~h}$. Serum calcium levels were grouped into the following categories: normal ( $\geq 8.7 \mathrm{mg} / \mathrm{dl})$, low $(7.5-8.6 \mathrm{mg} / \mathrm{dl})$, and critically low $(<7.5 \mathrm{mg} / \mathrm{dl})$. Ionized calcium levels (mmol/ 1) were converted to total calcium levels $(\mathrm{mg} / \mathrm{dl})$ by multiplying by a factor of 8 . This conversion yielded an identical, consistent reference range and was valid in a normoalbuminemic patient cohort undergoing elective surgery. Finally, postoperative PTH levels, obtained routinely only in the algorithm group, were recorded and
Fig. 1 Clinical pathway for postoperative calcium management following total thyroidectomy

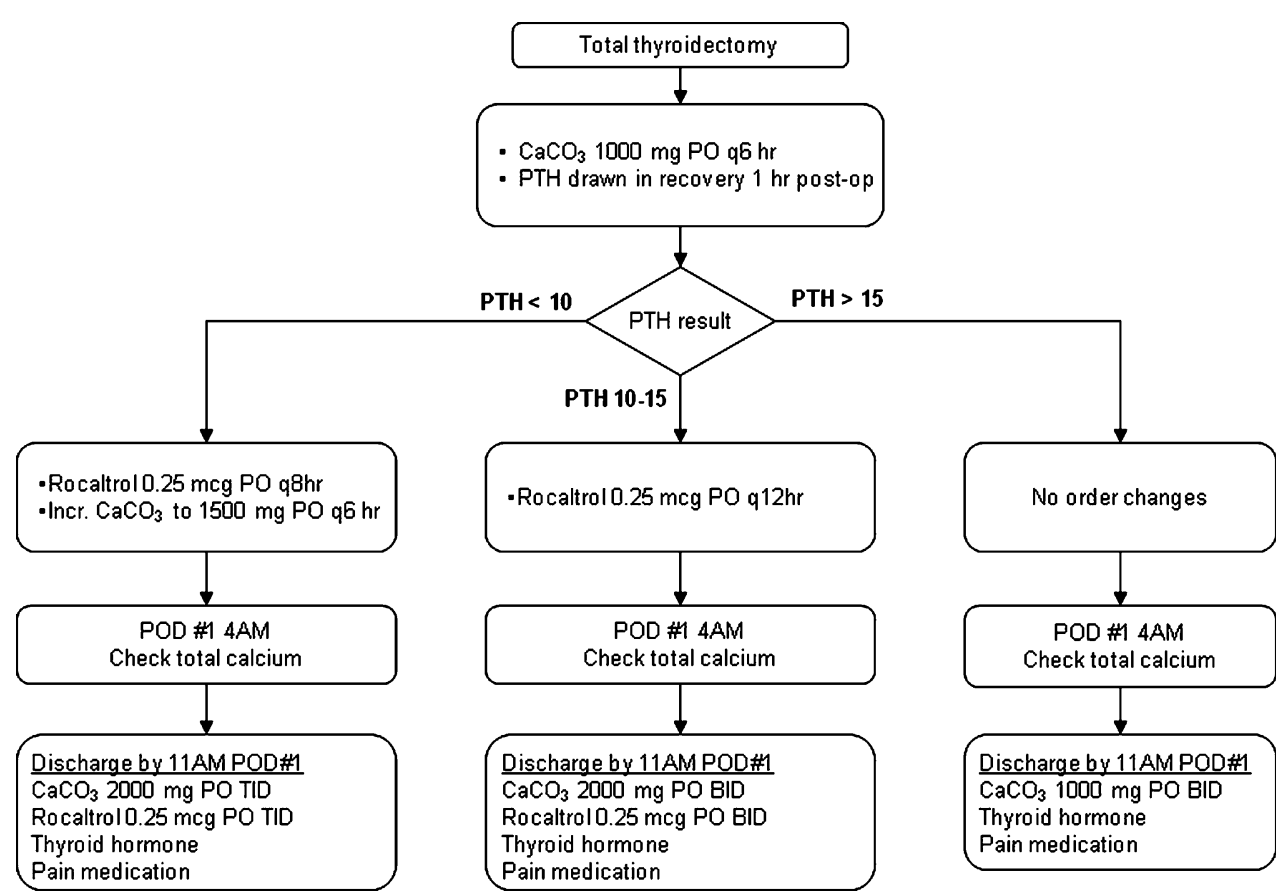


categorized as being normal ( $>15 \mathrm{pg} / \mathrm{ml})$, low-normal (10$15 \mathrm{pg} / \mathrm{ml})$, or low $(<10 \mathrm{pg} / \mathrm{ml})$.

Data analysis

Data were analyzed by Student's $t$-test, contingency table analysis, or one-way analysis of variance (ANOVA) for univariate analyses. Logistic regression was applied for a multivariate analysis. Differences were considered statistically significant for $p$ values $<0.05$. Data were analyzed with STATA/SE version 9.2 (StataCorp, College Station, TX).

\section{Results}

The algorithm was applied in 135 patients, and 288 patients were managed conventionally. Conventionally managed patients received calcium replacement in response to serially drawn serum calcium levels and/or clinical manifestations of hypocalcemia. Demographic characteristics of the comparison groups are shown in Table 1. Length of hospitalization was significantly shorter in the algorithm population $\quad(1.10 \pm 0.48$ days $\quad$ vs. $2.72 \pm 2.1$ days; $p<0.0001)$. When the indication for surgery was considered, non-algorithm patients were more likely to undergo surgery for thyroid cancer $(56.2 \%$ vs. $45.1 \%$; $p<0.05)$. Algorithm patients were less likely to have critically low calcium levels during hospitalization (Fig. 2). Of the total cases during the study period, $91 \%$ were managed by 5 surgeons, all of whom did more than 35 operations. The mean number of episodes of critical hypocalcemia was not significantly different when comparing high-volume $(>35$ cases) to low-volume ( $<35$ cases) surgeons (0.229 vs. $0.230 ; p=0.51)$. The protective impact of the algorithm was more pronounced among patients undergoing TTx for cancer, $30 \%$ of whom developed critically low calcium

Table 1 Demographic comparison of the study samples

\begin{tabular}{|c|c|c|c|}
\hline & $\begin{array}{l}\text { Non-algorithm } \\
(n=288)\end{array}$ & $\begin{array}{l}\text { Algorithm } \\
(n=133)\end{array}$ & $p$ Value \\
\hline Mean age \pm SD & $50.0 \pm 15.3$ & $47.6 \pm 15.7$ & 0.14 \\
\hline Female $(\%)$ & $223(77.4)$ & $112(84.2)$ & 0.10 \\
\hline \multicolumn{4}{|l|}{ Race/ethnicity (\%) } \\
\hline White & $191(66.3)$ & $83(62.4)$ & $<0.05$ \\
\hline Asian/Pacific Islander & $25(8.7)$ & $27(20.3)$ & \\
\hline Hispanic & $26(9.0)$ & $10(7.5)$ & \\
\hline Black & $20(6.9)$ & $7(5.3)$ & \\
\hline Native American/other & $26(9.0)$ & $6(4.5)$ & \\
\hline $\begin{array}{c}\text { Mean Charlson } \\
\text { co-morbidity } \\
\text { index } \pm \text { SD }\end{array}$ & $0.15 \pm 0.40$ & $0.13 \pm 0.54$ & 0.64 \\
\hline
\end{tabular}

levels when managed conventionally. In a multivariate logistic regression controlling for age, sex, race, insurance status (Medicare vs. private), co-morbidity (excluding thyroid cancer), length of hospitalization, and thyroid cancer status, only algorithm status predicted risk of having at least one low or critically low calcium value. Specifically, non-algorithm patients had a $2.17 \times$ greater risk of having a low calcium value during hospitalization compared with algorithm patients (95\% CI 1.20-3.92; $p<0.01)$.

Non-algorithm and algorithm patients were equally likely to receive IV calcium (14.9\% vs. $12.8 \%$; NS). Among patients who received IV calcium, non-algorithm patients received a higher average number of doses (1.86 vs. $1.29 ; p<0.05)$.

In algorithm patients, the 1-h PTH level correlated positively with the morning calcium level on postoperative day 1 (Fig. 3). Given the PTH cut-points assigned to different branches of the algorithm, we also examined the relationship between the morning calcium level and the PTH category (normal, low-normal, low; Fig. 4). Twentyone percent (28/133) of algorithm patients had low 1-h PTH levels, of which $28.6 \%$ (8/28) developed critically low calcium levels. Sixty-one percent (81/133) had normal $1 \mathrm{~h}$ PTH levels, with $5 \%(4 / 81)$ of those individuals developing critically low serum calcium. A low postoperative PTH level predicted low $(n=45)$ or critically low $(n=8)$ postoperative calcium levels with a sensitivity of $93 \%$ and a specificity of $98 \%$. A normal 1-h PTH was associated with higher mean calcium compared with either the low-normal or low PTH groups $(p<0.0001)$. Calcium values in the low versus low-normal PTH groups were not significantly different.

\section{Discussion}

The use of intraoperative or postoperative PTH levels to predict hypocalcemia following TTx remains controversial. Several groups have found that PTH levels cannot reliably predict postoperative hypocalcemia [14, 15]. Lombardi et al. [16] reported that parathyroid hormone measurements less than $10 \mathrm{pg} / \mathrm{ml}$ levels alone $4 \mathrm{~h}$ after surgery were unable to accurately predict hypocalcemia in $13.4 \%$ of their study population, $2.1 \%$ of whom were symptomatic. Other investigators support the use of PTH in identifying patients likely to develop hypocalcemia following thyroidectomy [5-13]. McLeod et al. [17] showed that postoperative PTH levels less than $12 \mathrm{pg} / \mathrm{ml}$ had a $100 \%$ sensitivity and $92 \%$ specificity for predicting hypocalcemia. This was corroborated by the work of Sywak et al. [18], which demonstrated a sensitivity of $90 \%$ and a specificity of $84 \%$ of PTH levels from $3-10 \mathrm{pg} / \mathrm{ml}$ drawn 
Fig. 2 Comparison of outcomes for all patients (a), those patients with a non-cancer diagnosis (b), and those with a cancer diagnosis (c). $* p<0.05 ; * * p<0.01$

$4 \mathrm{~h}$ after surgery for predicting postoperative hypocalcemia (as defined by corrected calcium levels $\leq 2.0 \mathrm{mmol} / \mathrm{l}$ ). Similarly, in a study looking at the experiences of five separate Australian institutions, Grodski and Serpell [19] demonstrated a sensitivity, specificity and positive predictive value of $70.7 \%, 92.6 \%$, and $71.6 \%$, respectively, for low PTH levels $(<10 \mathrm{ng} / \mathrm{l}) 4 \mathrm{~h}$ after surgery in predicting postoperative hypocalcemia $(<2.00 \mathrm{mmol} / \mathrm{l})$. This led to the inclusion of the routine measurement of PTH postoperatively in the Australian Endocrine Surgeons Guidelines for patients undergoing total thyroidectomy [20]. In a 2008 meta-analysis of 27 papers, Grodski and Serpell [19] reiterated this recommendation, reporting that postoperative PTH at any time within $24 \mathrm{~h}$ of total thyroidectomy can be used to accurately predict the development of hypocalcemia and anticipate the need for calcium replacement, albeit with class $\mathrm{C}$ evidence. In this same vein, our findings support the use of PTH to identify those patients in whom postoperative hypocalcemia may be anticipated.

Our practice is to administer oral calcitriol to all patients with low and low-normal postoperative PTH levels. The efficacy of this drug treatment is supported by a number of previous studies [21-23]. Tartaglia et al. [24] showed in 2005 that oral administration of calcium and calcitriol after thyroidectomy significantly decreases the risk of postoperative hypocalcemia. Calcitriol induces a fourfold increase in the uptake of oral calcium [25], reducing by half the dosage required to maintain physiologic levels in the postoperative period. This requires less frequent dosing and alleviates much of the nausea and constipation associated with large amounts of oral calcium, ultimately leading to superior patient compliance. Additionally, since a generic form became available in 2001, calcitriol is inexpensive ( $\$ 1.20$ per $0.25 \mathrm{mcg}$ pill, TEVA Pharmaceuticals, Petach Tikva, Israel). When used judiciously in a sliding scale fashion, calcitriol does not lead to overtreatment (to date, this practice has not resulted in any episodes of hypercalcemia in our experience). For these reasons, we emphasize the use of calcitriol in combination with lower doses of oral calcium in lieu of large doses of oral calcium alone. In a separate but related study, we found that algorithmic management results in a significantly decreased length of hospital stay, reduced costs associated with multiple lab draws, and a reduced need for IV calcium administration. Furthermore, implementation of the algorithm reduced the number of hospital readmissions for postoperative complications to zero. These combined benefits yielded an average savings of $\$ 1,631$ (74\%) per patient in our hospital system [26].
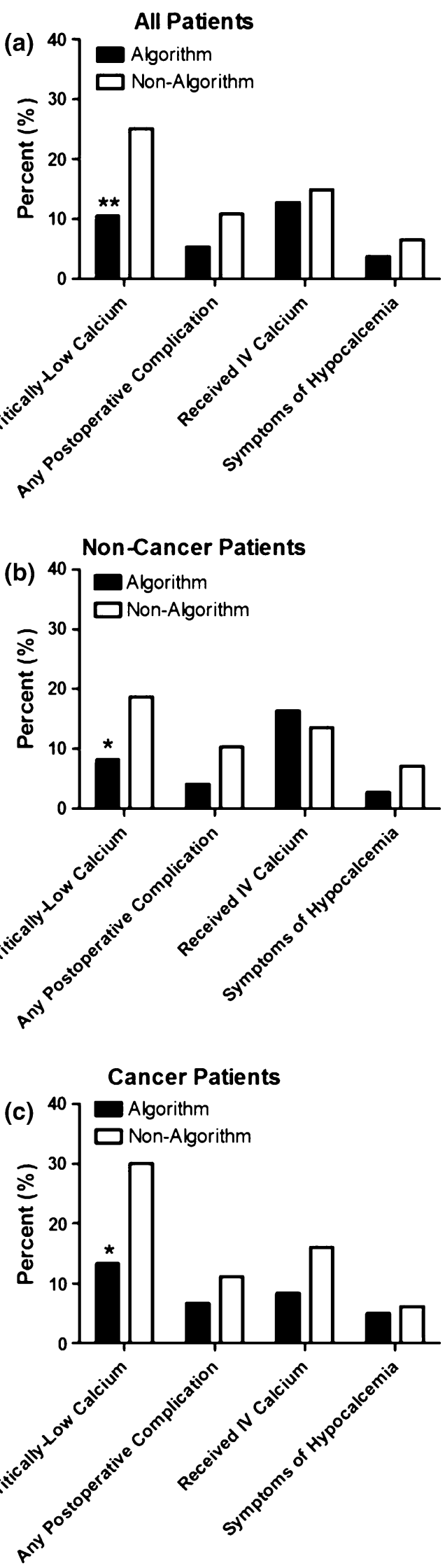


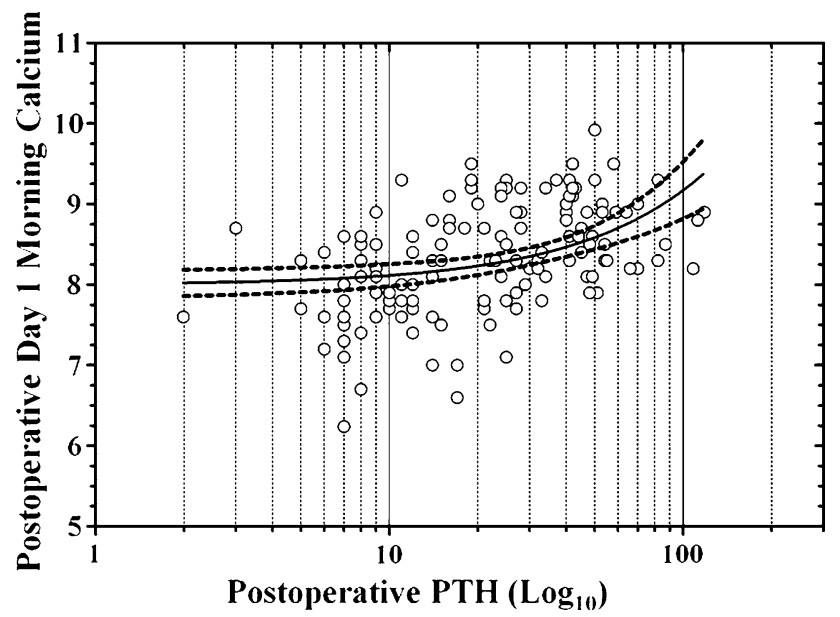

Fig. 3 Scatter graph demonstrating the relationship between postoperative day 1 calcium and 1-h PTH

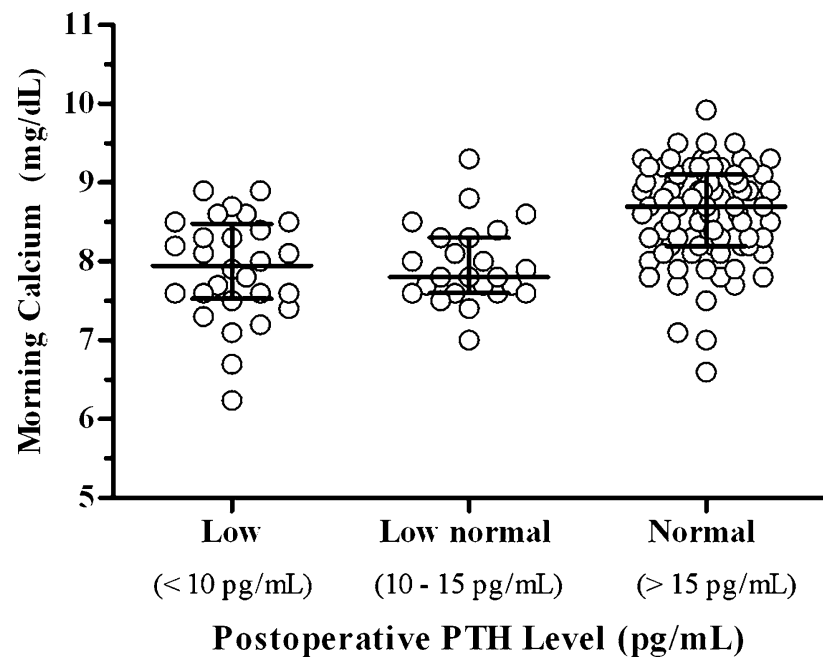

Fig. 4 Scatter graph demonstrating the relationship between postoperative day 1 calcium and 1-h PTH grouped by measurement range

We noted a statistically significant difference in the percentages of patients undergoing TTx for a cancer versus non-cancer diagnosis between the two study samples. Given the need to conduct a more aggressive operation in the treatment of malignancy, we postulated that an increased frequency of postoperative hypoparathyroidism would be observed in this patient population. The hypothesis was borne out in our data, which demonstrated a greater prevalence of critically low calcium levels among patients undergoing TTx for cancer, with a rate of $30 \%$ in those managed conventionally. This increased risk was present regardless of postoperative management, suggesting that, at present, our algorithm may be not aggressive enough to prevent hypocalcemia in this patient subset. In a randomized analysis of patients undergoing TTx with central lymph node dissection for papillary cancer, Roh et al. [22] demonstrated a postoperative hypocalcemia
$(<8.0 \mathrm{mg} / \mathrm{dl})$ rate of $44 \%$ in patients who received neither oral calcium supplementation nor calcitriol. This rate decreased to $24 \%$ with administration of oral calcium, and $8.2 \%$ with a combination of oral calcium and calcitriol. The implication is that patients undergoing TTx for cancer are particularly vulnerable to postoperative hypoparathyroidism and anticipatory measures should be routinely employed.

The major limitation of postoperative PTH measurement is the possibility that these values may be spuriously normal or high in a patient that subsequently develops unanticipated critically low calcium levels. Among the 133 algorithm patients in our study, 81 (61\%) had normal 1-h PTH levels, and only 5\% (4/81) went on to develop critically low morning calcium levels. Therefore, though we acknowledge that spuriously normal PTH levels are occasionally found, these occurrences are rare. Notably, appropriate and timely intervention was taken in all algorithm patients who ultimately required IV calcium supplementation. The routine measurement of serum calcium on the morning following operation is an additional safeguard that permits identification of patients with hypoparathyroidism, even among those not correctly identified by the postoperative PTH level. Furthermore, all patients are discharged on continued calcium supplementation for 2 weeks, regardless of inpatient lab results (a low dose of $1,000 \mathrm{mg}$ calcium carbonate twice daily with no calcitriol is used for patients with completely normal PTH and calcium levels). This affords a third tier of protection against the development of hypocalcemia should it occur in a delayed fashion following discharge, thus preventing hospital readmissions.

This study is limited by its retrospective, nonrandomized design. Despite these weaknesses, our data favor implementation of an algorithmic approach to calcium management after total thyroidectomy that is informed by measurement of postoperative PTH level.

Acknowledgments The authors are grateful to the Earl Gales Family Foundation for their support of this project. The study was conducted with the approval of the UCLA institutional review board, protocol \# G08-11-016-01.

Open Access This article is distributed under the terms of the Creative Commons Attribution Noncommercial License which permits any noncommercial use, distribution, and reproduction in any medium, provided the original author(s) and source are credited.

\section{References}

1. Bergenfelz A, Jansson S, Kristoffersson A et al (2008) Complications to thyroid surgery: results as reported in a database from a multicenter audit comprising 3, 660 patients. Langenbeck's Arch Surg 393:667-673 
2. Glinoer D, Andry G, Chantrain G et al (2000) Clinical aspects of early and late hypocalcaemia afterthyroid surgery. Eur J Surg Oncol 26:571-577

3. Demeester-Mirkine N, Hooghe L, Van Geertruyden J et al (1992) Hypocalcemia after thyroidectomy. Arch Surg 127:854-858

4. Bellamy R, Kendall-Taylor P (1995) Unrecognized hypocalcaemia diagnosed 36 years after thyroidectomy. J R Soc Med 88:690-691

5. Lindblom P, Westerdahl J, Bergenfelz A (2002) Low parathyroid hormone levels after thyroid surgery: a feasible predictor of hypocalcemia. Surgery 131:515-520

6. Richards ML, Bingener-Casey J, Pierce D et al (2003) Intraoperative parathyroid hormone assay: an accurate predictor of symptomatic hypocalcemia following thyroidectomy. Arch Surg 138:632-636

7. Lombardi CP, Raffaelli M, Princi P et al (2004) Early prediction of postthyroidectomy hypocalcemia by one single iPTH measurement. Surgery 136:1236-1241

8. Vescan A, Witterick I, Freeman J (2005) Parathyroid hormone as a predictor of hypocalcemia after thyroidectomy. Laryngoscope 115:2105-2108

9. Barczyński M, Cichoń S, Konturek A et al (2008) Applicability of intraoperative parathyroid hormone assay during total thyroidectomy as a guide for the surgeon to selective parathyroid tissue autotransplantation. World J Surg 32:822-828

10. Ghaheri BA, Liebler SL, Andersen PE et al (2006) Perioperative parathyroid hormone levels in thyroid surgery. Laryngoscope 116:518-521

11. Fabio F, Casella C, Bugari G et al (2006) Identification of patients at low risk for thyroidectomy-related hypocalcemia by intraoperative quick PTH. World J Surg 30:1428-1433

12. Gentileschi P, Gacek I, Manzelli A et al (2008) Early (1 hour) post-operative parathyroid hormone $(\mathrm{PTH})$ measurement predicts hypocalcaemia after thyroidectomy: a prospective case-control single-institution study. Chir Ital 60:519-528

13. Wong C, Price S, Scott-Coombes D (2006) Hypocalcaemia and parathyroid hormone assay following total thyroidectomy: predicting the future. World J Surg 30:825-832

14. Ghaheri BA, Liebler SL, Andersen PE et al (2006) Perioperative parathyroid hormone levels in thyroid surgery. Laryngoscope $116: 518-521$
15. Starr FL, DeCresce R, Prinz RA (2000) Normalization of intraoperative parathyroid hormone does not predict normal postoperative parathyroid hormone levels. Surgery 128:930-936

16. Lombardi CP, Raffaelli M, Princi P et al (2006) Parathyroid hormone levels 4 hours after surgery do not accurately predict post-thyroidectomy hypocalcemia. Surgery 140:1016-1025

17. McLeod IK, Arciero C, Noordzij JP et al (2006) The use of rapid parathyroid hormone assay in predicting postoperative hypocalcemia after total or completion thyroidectomy. Thyroid 16:259

18. Sywak MS, Palazzo FF, Yeh M et al (2007) Parathyroid hormone assay predicts hypocalcaemia after total thyroidectomy. Aust N Z J Surg 77:667-670

19. Grodski S, Serpell J (2008) Evidence for the role of perioperative PTH measurement after total thyroidectomy as a predictor of hypocalcemia. World J Surg 32:1367-1373

20. Australian Endocrine Surgeons Guidelines AES06/01 Group (2007) Postoperative parathyroid hormone measurement and early discharge after total thyroidectomy: analysis of Australian data and managment recommendations. Aust N Z J Surg 77:199202

21. Bellantone R, Lombardi CP, Raffaelli M et al (2002) Is routine supplementation therapy (calcium and vitamin D) useful after total thyroidectomy? Surgery 132:1109-1113

22. Roh J-L, Park J-Y, Park C-I (2009) Prevention of postoperative hypocalcemia with routine oral calcium and vitamin D supplements in patients with differentiated papillary thyroid carcinoma undergoing total thyroidectomy plus central neck dissection. Cancer 115:251-258

23. Roh J-L, Park C-I (2006) Routine oral calcium and vitamin D supplements for prevention of hypocalcemia after total thyroidectomy. Am J Surg 192:675-678

24. Tartaglia F, Giuliani A, Sgueglia M et al (2005) Randomized study on oral administration of calcitriol to prevent symptomatic hypocalcemia after total thyroidectomy. Am J Surg 190:424-429

25. Holick MF (2007) Vitamin D deficiency. N Engl J Med 357:266281

26. Kulkarni RP, Ituarte PHG, Gunderson D et al (2008) Clinical pathways improve hospital resource utilization in endocrine surgery. American College of Surgeons Clinical Congress, San Francisco, October 2008 (poster) 